Cahiers de recherches médiévales

\title{
Narcisse à la fontaine : du « conte » à « l'exemple »
}

\section{Emmanuèle Baumgartner}

\section{(2) OpenEdition}

Journals

Édition électronique

URL : https://journals.openedition.org/crm/70

DOI : $10.4000 / \mathrm{crm} .70$

ISSN : $1955-2424$

Éditeur

Honoré Champion

Édition imprimée

Date de publication : 10 décembre 2002

ISSN : 1272-9752

Référence électronique

Emmanuèle Baumgartner, « Narcisse à la fontaine : du « conte » à « l'exemple » », Cahiers de recherches médiévales [En ligne], 9 | 2002, mis en ligne le 05 janvier 2007, consulté le 15 décembre 2022. URL : http://journals.openedition.org/crm/70 ; DOI : https://doi.org/10.4000/crm.70

Ce document a été généré automatiquement le 15 décembre 2022.

Tous droits réservés 


\title{
Narcisse à la fontaine : du « conte » à « l'exemple »
}

\author{
Emmanuèle Baumgartner
}

1 Le lai ou le conte de Narcisse, la plus ancienne version française du récit d'Ovide et la plus infidèle, compte environ 1000 vers. La séquence que Christine de Pizan consacre à Narcisse dans L'Epistre Othea, au début du XV ${ }^{\mathrm{e}}$ siècle, intitulée Narcisus qui se mira en la fontaine, comporte 25 lignes, texte, glose et allégorie compris ${ }^{1}$. La différence quantitative, telle quelle, ne signifie rien, mais elle rend bien compte du statut acquis par le héros d'Ovide et son histoire qui, tel est le propre du récit mythique et de sa plasticité, peuvent aussi bien donner matière à des versions plus ou moins développées qu'à des allusions et citations immédiatement reconnues. La comparaison avec Narcisse, le jeune homme épris d'une ombre vaine et fasciné par la beauté qui en émane, est récurrente chez les troubadours, chez les trouvères et dans le roman médiéval pour dire l'impossible étreinte de l'amant et ce n'est pas le lieu d'en reprendre l'inventaire ${ }^{2}$. Je voudrais plus spécifiquement montrer à l'aide de quelques exemples échelonnés dans le temps comment Narcisse et la fontaine où il «mira sa face et ses ieux vers » ont fait l'objet du XII ${ }^{e}$ à la fin du XIV ${ }^{e}$ siècle d'approches et d'usages très divers en dépit de la remarquable permanence du scénario ovidien. Telle est d'ailleurs l'une des conditions de survie (ou de mort) d'un mythe que de pouvoir satisfaire d'autres attentes, dans d'autres cultures, pour d'autres publics ${ }^{3}$.

D'une manière générale, les écrivains du Moyen Âge français n'ont jamais retrouvé les secrets perdus de la poésie d'Ovide, eux qui ont dû lutter avec le rythme grêle du couplet d'octosyllabes, avec une syntaxe trop contrainte et trop raide et plus encore avec un lexique encore rudimentaire, et qui ignorent ou presque l'art de la métaphore. Quant à leurs lectures de Narcisse, elles relèvent toutes, à des profondeurs diverses, de la moralisation, au sens médiéval du terme bien entendu, d'une recherche de l'utilitas, de l'exemplarité au premier degré, bien étrangère au poète latin. "L'exemple de Narcisse ", rappelle Jean Frappier ", doit mettre en garde à la fois contre l'orgueil et contre les folies de l'amour $»^{4}$. Sans doute était-ce aussi le prix à payer pour adapter et donner à lire les Métamorphoses, cette "bible du paganisme ", et plus encore la 
troublante histoire d'un être qui représente très tôt le repliement sur soi, le refus de tout pacte avec l'autre, de tout contact charnel, le retrait du monde pour la pure contemplation de soi et l'absolue fascination que peut exercer la beauté. Avant de comprendre qu'il se désire lui-même, le Narcisse d'Ovide s'éprend d'abord de la merveilleuse statue taillée dans le marbre de Paphos dont l'eau lui renvoie l'image admirable... Serait-ce parce qu'ils ont récusé ce sens mais parce que Narcisse, figure condamnable, reste au Moyen Âge toujours désirable, que les écrivains médiévaux ont peu à peu dévié le cours du récit mythique, préféré la source à l'ombre qu'elle reflète, écarté le héros pour redonner vie à une «fontaine » dont le cours retrouvé a irrigué en profondeur le texte médiéval, est devenu la source vive d'une réflexion sur la nature et les effets de l'amour?

Le premier à subvertir le sens du mythe d'une manière si radicale qu'elle n'a pas eu de suite est l'auteur anonyme du Lai de Narcisse. Substituer Dané, la fille de roi avide d'aimer et de s'unir à celui qu'elle désire, à la triste Echo, c'est faire plus que d'inscrire le mythe dans un contexte médiéval et un cadre féodal. Ce qui entre alors en jeu, c'est de montrer la conversion de Narcisse, même in extremis, à l'amour charnel, loi de Nature. Qu'il reconnaisse son erreur, et le texte prend valeur d'exemple. C'est là une dimension totalement absente du texte ovidien selon lequel Narcisse aux enfers, bourreau endurci de lui-même, "se regardait encore dans l'eau du Styx ". L'auteur médiéval au contraire donne à la fois à Narcisse la possibilité de se tourner vers l'autre, vers une jeune fille aimable et qui crie (peut crier, à la différence d'Echo) son droit à être aimée, et il lui fait découvrir juste avant de mourir (le mythe résiste)l'échange des regards et l'étreinte des corps, à défaut de l'échange des mots d'amour. Comprenant qu'il s'est condamné à mourir pour s'être refusé à aimer

Que, se m'amors seüst $\mathrm{u}$ prendre

Et je veïsse autrui que moi,

Ne fuisce pas en tel esfroi.

Dix, s'or venoit par aventure [il s'agit de Dané],

Ja porroit bien estre seüre

Que ele conquerroit m'amor

Et me geteroit de langor.

Bien me devoit max avenir

Quant onques ne le voil oïr.(vv. 960-968)6

le jeune homme, lorsque Dané le rejoint, la regarde sans pouvoir parler, mais

La fontaine li mostre au doit

Et l'onbre qui si le deçoit.

Les bras li tent, les levres muet,

Les ex ovre si com il puet ;

Sanblant li fait que se repent (vv. 979-983).

Et lorsque Narcisse meurt, Dané, comme Iseut, le rejoint dans la mort.

Cependant, alors qu'il a ainsi détourné et banalisé le mythe et fait de Narcisse un «essample» de la puissance de l'amour à laquelle chaque être doit se soumettre sous peine de mort, l'auteur du Lai est resté beaucoup plus proche d'Ovide dans la description qu'il donne de l'arrivée du héros à la «fontaine» et de la fontaine ellemême. Il a ainsi fixé pour des générations d'écrivains les grandes lignes du scénario « arrivée au bord de la fontaine » et de la description du lieu. Comme point de départ, la chasse d'un cerf qui n'est pas encore blanc ${ }^{7}$, mais qui trompe tout autant les efforts du chasseur; la soif qui brûle le héros, à l'heure fatidique de midi ; la découverte d'une eau 
claire, douce et bonne à boire; le lieu propice, aménagé par l'homme, la margelle de marbre se substituant à la source naturelle du poète latin; l'herbe drue qui invite à s'allonger; l'arbre pour attacher le cheval. Sont ici réunis, pour la première fois exprimés en langue française, tous les traits et les attraits du lieu fontaine, qui pourra aussi bien se transformer en lieu mortifère qu'en lieu érotisé de la rencontre avec la fée, de la plainte amoureuse, de l'invention poétique. Les exemples dans la littérature ultérieure sont innombrables. On se contentera de citer en écho immédiat quelques vers du Roman de Troie, texte à peu près contemporain, qui ouvre lui aussi le récit mythique du Jugement de Pâris sur le motif de la chasse interrompue, de la chaleur extrême, de l'absence de vent (trait repris à Ovide), de l'arrivée du chasseur au bord d'une fontaine où personne, jamais, ne s'est abreuvée, le sommeil de Pâris, propice à l'apparition des déesses, se substituant alors à la sidération mortifère de Narcisse.

L'autrier, es kalendes de mai

Chacöe en Inde la menor

Un cerf, ce m'est vis, correor.

Le jor le chacierent mi chien ;

Assez corui, ainc n'en pris rien.

Mout fist grant chaut d'estrange guise,

Ne venta gaires le jor bise.

Mes veneors e toz mes chiens

Perdi el val de Citariens.

Lez la funtaine ou riens n'abeivre,

De desoz l'onbre d'un geneivre

M'estut dormir, nel poi müer. (vv. 3860-3871) ${ }^{8}$

On retiendra de cette première série de textes deux éléments : l'auteur du Lai de Narcisse n'a pas conservé l'absence de vent, qui explique chez Ovide la durable immobilité de l'eau, de l'image qu'elle reflète sans trouble.

Si la feuille éperdue effleure la napée,

Elle suffit à rompre un univers dormant... ${ }^{9}$

Le choix du récit médiéval est en effet d'en finir avec l'enchantement de Narcisse, de brouiller la surface immobile de la fontaine -c'est là le rôle de Dané- pour détourner le jeune homme de la contemplation de soi. Il est également remarquable que dans ces deux textes le lieu fontaine tisse un premier écho entre Narcisse et Pâris, devient le lieu où peuvent aussi bien s'accomplir un destin de mort, dans le refus de l'aventure amoureuse, que le choix de Vénus, la révélation de la puissance vitale de l'amour.

Dans le roman arthurien en vers ou en prose, Narcisse n'est guère que le nom d'un amour impossible et il n'en est fait que de brèves mentions, qui attestent au reste de la diffusion de l'histoire ${ }^{10}$. Mais est-il nécessaire d'évoquer la récupération par Chrétien de Troyes du lieu fontaine dans le Chevalier au Lion, et sa fortune ultérieure: un lieu où passer à travers le miroir, un lieu où se noue la destinée du chevalier amant, où se dit ensuite, et d'abord dans le Tristan en prose, la joie et plus souvent la douleur d'aimer sans espoir? Si on en reste cependant à la filiation directe, le texte majeur est bien entendu le Roman de la Rose, qui reprend assez fidèlement le récit d'Ovide-Echo fait retour- tout en déplaçant définitivement la charge mythique de Narcisse à la fontaine. Le narrateur expose assez longuement, dans un excursus hors- rêve, le cas Narcisse et en tire une morale qui a pu déconcerter, mais qui s'applique assez bien, somme toute, aux préoccupations à venir de l'amant: ne pas être victime de l'indifférence de la 
femme aimée. Quant au rêveur, qui identifie la «fontaine au pin», soigneusement agencée par Nature, comme lieu de la mort de Narcisse,

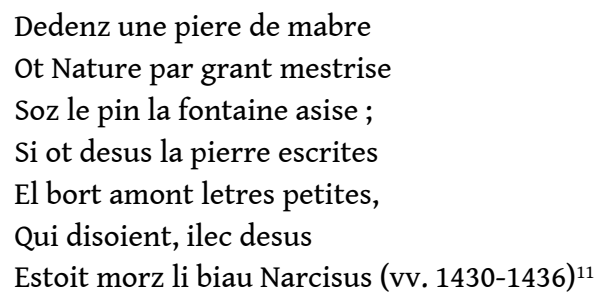

il a tôt fait de renvoyer aux oubliettes de la mythologie le drame de Narcisse et de se mirer dans l'eau de la fontaine pour y découvrir l'objet de sa quête d'amour. Par la grâce du rêve, la fontaine, il est vrai, a été le lieu, du texte latin au texte médiéval, d'une métamorphose inattendue. Lieu de mort dans le discours «ovidien» du narrateur, qui s'est bien gardé de rappeler la métamorphose du corps en narcisse, elle est devenue à l'approche du rêveur un lieu de vie, sous le double signe, mais n'est-ce pas le même?, d'Amour et de Nature qui a mis tout son art à la créer (vv. 1431-1432). L'eau immobile « court » désormais, coule à grands flots, toujours fresche et novele. Grâce à elle, l'herbe pousse en toutes saisons, toujours épaisse et drue. A l'unique reflet de Narcisse se substitue la vision du buisson de roses et de l'estre d'un vergier dont lecteur et rêveur connaissent déjà la luxuriance, la fertilité et l'abondance des ressources érotiques. A la contemplation de l'ombre se substitue le regard orienté vers l'autre d'un jeune homme éperdu, immédiatement pénétré jusqu'au fond du cœur par la douce saveur des roses. ${ }^{12}$ Le narrateur sans doute annonce aussitôt l'échec subi dans la «réalité » (vv. 1606-1612)et le caractère déceptif de ce nouveau miroir. Du moins le rêveur a-t-il tenté l'aventure amoureuse, pris le risque de traverser le miroir périlleux. Désormais, en l'effacement conscient mais provisoire de l'histoire de Narcisse, c'est à la fontaine où le rêveur se garde bien d'apaiser sa soif que se noue le destin, la rage d'aimer.

11 Jean de Meun au reste a pris acte de ce déplacement. Genius dans son sermon ne met pas vraiment en balance l'exemple de Narcisse et celui de Pygmalion ${ }^{13}$. C'est la fontaine recréée par Guillaume de Lorris au sein du verger de Deduit, une fontaine qui reste sans doute un lieu de péril puisqu'elle "tua le biau Narcisus/qant il se miroit iqui sus" (vv. 20381-2), qu'il oppose à la plénitude féconde de la Fontaine de vie qui irrigue le Parc du sage berger. Et les auteurs qui s'inscrivent à partir du XIVe siècle dans le sillage du Roman de la Rose s'intéressent tout autant sinon plus à la Fontaine d'Amors qu'à l'histoire de Narcisse.

Il n'est guère, pour ne pas pratiquer ce transfert et même l'évacuer, que l'auteur de l'Ovide moralisé qui, tout en restant très fidèle au texte d'Ovide ${ }^{14}$, ne s'interdit pas cependant de faire quelques emprunts habilement trafiqués au texte de Guillaume de Lorris ${ }^{15}$. Comme à son habitude, l'auteur découpe pour les besoins de la glose le récit d'Ovide en commentant au fur et à mesure la signification d'Echo, de Narcisse et de la fontaine «decevable», et en donne une interprétation allégorique très prévisible. Echo "denote bone renomee» (v.1465); Narcisse est la figure emblématique des «folz musors de sens voidiez,/ des orgueilleus, des sorcuidiez,/ qui des biens temporeus abusent, /qui se mirent et qui s'amusent/ aus faulz mireoirs de cest monde" (vv. 1904-1909). Le traitement réservé à la fontaine est plus original. Si l'auteur la caractérise d'abord et sans grande invention comme «faulz mirooir» (v.1761) ou 
comme «fontaine decevable/ Qui fet l'ombre fainte et muable/ Cuidier vrai bien et parmanant » (vv. 1933-1935), il modifie ailleurs une indication du Roman de la Rose qu'il combine avec le texte d'Ovide. Après avoir en effet rappelé comment Nature, par sa maistrise, créa la fleur narcisse (et non la fontaine elle-même, comme chez Guillaume de Lorris), il précise, bloquant ainsi la «métamorphose » de la fontaine en Fontaine d'amors, invention particulièrement novatrice, on l'a rappelé, de Guillaume de Lorris :

De teulz flours est toute porprise

La fontaine ou cil, par folie,

En soi mirant perdi la vie.

Puis il ajoute, pour faire bonne mesure, une glose «étiologique ${ }^{16}$ :

Dou non Narcisus est nomee

La fontaine et bien renomee,

S'a non 'fontaine Narcisi'.

La flours et la vile autresi,

Apele on Narci, qui ore est

La ou fu jadis la forest

Ou la fontaine estoit assise (vv. 1844-1853),

décidant ainsi de la métamorphose en ville de la fontaine jadis enclose dans la forêt et à tout jamais tarie.

On sait l'importance qu'a eue à partir du XIV e siècle l'ovide moralisé pour la diffusion des Métamorphoses et comment il a été une sorte de réservoir où écrivains et poètes des $\mathrm{XIV}^{\mathrm{e}}$ et $\mathrm{XV}^{\mathrm{e}}$ siècles ont puisé des «fictions» qu'ils ont plus ou moins fidèlement réécrites, sans trop s'embarrasser bien souvent de la glose ${ }^{17}$. Il semble cependant que le mythe de Narcisse a en partie échappé à la lourde chape de plomb que jette sur lui l'auteur de l'Ovide moralisé et que ce sont très directement le Roman de la Rose et la métamorphose qu'il fait subir à la fontaine qui ont inspiré deux auteurs sur lesquels j'achèverai ce bref parcours : l'auteur des Échecs amoureux (en vers) et Evrard de Conty, auteur à la fin du XIV e siècle d'un texte en prose, Le Livre des Eschez amoureux moralisés, qui selon son auteur, «fut fait et ordené principalment a l'instance d'un autre, fait en rimes nagueres, et de nouvel venu a congnoissance, qui est intitulé 'Des Eschez amoureux' ou 'Des Eschez d'amours', aussi come pour declairier aucunes choses qui semblent estre obscures et estranges de prime face $»{ }^{18}$

Le rêveur qui refait dans les Échecs amoureux en vers le chemin naguère parcouru par le rêveur du Roman de la Rose et en reconnaît en promeneur consciencieux les points de passages obligés, arrive à son tour à la fontaine « amoureuse/dangereuse » (vv. 713-714) contre laquelle l'a déjà soigneusement mis en garde la chaste Diane. Mais, nouveau Pâris, le rêveur a fait son choix : pour avoir préféré Vénus, servante de Nature, à Junon et à Pallas, il a reçu de la déesse l'assurance de posséder une dame cent fois plus belle qu'Hélène...et il a donc rejeté l'enseignement de Diane, la voie sévère de la continence. Une fois introduit comme son prédécesseur dans le verger de Deduit, le rêveur découvre en un «trop biau lieu (v. 708) la fontaine amoureuse ;/ Ch'est la fontaine dangereuse, / Que Dÿane tant me blasma, / Ou Narchisus son ombre ama,/ Mais non contrestant sa parole,/ Qui ne me sambla que frivole, / Je me tray pres de la fontaine» (vv. 713-719). Il voit alors, comme dans le Roman de la Rose, «les lettres petites/ Sur le bort de la pierre escrites,/ Qui seignifioient comment/ Narchisus dolereusement/ Delés la fontaine moru» (vv.725-729). Mais le jeune homme a tôt fait de répartir les torts, de se persuader que Narcisse est mort «par son oultraige/ ou par aucune grant folie/ et que 
la fontaine jolie/ n'en devoit pour ce blasme avoir » (vv. 740-743). Rien ne peut donc le détourner de «chelle fontaine joyeuse / qui lui sambla si tres gracieuse » et de s'y mirer assez longuement pour apercevoir enfin au miroir de la fontaine dont les cristaux reflètent alternativement chaque moitié du verger, non le buisson de roses, devant lequel il a déjà passé, mais le lieu où se sont retirés Deduit, le dieu d'Amour et leur compagnons, qu'il s'empresse de rejoindre.

Finablement tant m'y miray,

Que chil bel cristal dechevant ${ }^{19}$

Me raporterent au devant

Le samblanche du dieu d'Amours,

Si com je l'os veü aillours,

Et de Deduit et de Leesche

Et de Biauté et de Jonesche

Et de toute lor compaignie

Gracïeuse et bien enseignie ; (vv. 820-828)

Un peu plus avant dans le texte, le dieu d'Amour confirme sur le mode hyperbolique les vertus de la fontaine / les vertus de l'amour, tout en condamnant Narcisse, qui n'a pas su garder mesure :

«Ch'est la fontaine merveilleuse

Qu'aucun appelent perilleuse

Par ygnorance ou par envie,

Car ch'est la fontaine de vie,

De pais, de joye et de plaisanche

Et de toute bonne esperanche,

Et se Narchisus y mouru,

Che fu pour ce qu'il y couru

Trop chaus, je le te chertefie,

Et qu'il but trop a une fie,

Car il n'i sçot tenir mesure,

Si que che n'est mie droiture

Que nulz la fontaine en encoupe,

Puis qu'il y moru par sa coupe.(vv. 2363-2375).

Comme dans le Roman de la Rose, Narcisse et son triste sort sont donc congédiés pour cause d'« outrage » ou de «demesure » . La fontaine, elle, à laquelle le jeune homme est invité à boire "par mesure» (v. 2380) reste bien le lieu où s'oriente et se décide un destin qui, quelle que soit l'issue de la seconde partie d'échecs ${ }^{20}$, doit d'abord passer par la «vie delitable» (vv. 2387-2388), " qu'il n'est vie si pourfitable ». Comme l'a naguère signalé P.-Y. Badel ${ }^{21}$, «la clé de l'œuvre est sans aucun doute le Jugement de Pâris ", l'auteur faisant des trois déesses, dans la tradition des mythographes, l'incarnation des trois étapes selon lesquelles doit se dérouler une vie idéale, de la vie voluptueuse à la vie contemplative (Pallas) en passant par la vie active (Junon). Ce qui représente déjà une nette amélioration du choix manichéen présenté au duc de Normandie Guillaume Longue-Épée par l'abbé Martin de Jumièges entre vie pratica et vie teorica ${ }^{22}$...On remarquera aussi comment la fontaine devient dans ce texte le lieu où se répondent et se hiérarchisent deux mythes de l'amour jusque là distincts ${ }^{23}$. Si Narcisse et son impardonnable «outrage», préférer le beau au vif, est bel et bien le perdant - et pour plusieurs siècles encore- il n'est plus que les ignorants ou les envieux pour rejeter le choix de Vénus et exclure les plaisirs charnels, pour traiter encore de "perilleuse » la fontaine «merveilleuse», aussi «gracieuse» que «delitable» à qui sait y boire sans toutefois perdre raison. 


$$
\begin{aligned}
& \text { Pygmalion, il déclare qu'il réserve à plus tard l'examen du cas de Narcisse. C'est en effet } \\
& \text { après avoir commenté, d'après les Échecs en vers, l'emprisonnement de Bel Accueil au } \\
& \text { château de Jalousie qu'Evrart insère en commentaire une description de «la fontaine } \\
& \text { Narcisus que aucuns appellent la fontaine d'amour et moult souvent aussi l'appelle on } \\
& \text { la fontaine perilleuse ", suivie d'un bref rappel de l'histoire de Narcisus (pp. 584-585). La } \\
& \text { description de la fontaine est une sorte de mise en prose assez fidèle du texte de } \\
& \text { Guillaume de Lorris, présenté comme «l'acteur qui en parle premiers aprés Ovide ", } \\
& \text { tout comme le résumé ensuite donné de l'histoire de Narcisse. Evrart de Conty a } \\
& \text { cependant repris pour le dénouement le texte d'Ovide: } \\
& \text { Et dit oultre la fable que son corps fut mués en une fleur qui encore est Narcisus } \\
& \text { appellee, et son ame fu translatee en enfer ou elle encore se mire et regarde sa face } \\
& \text { es palus infernauls ou elle se esmerveille de sa beauté.(p. 586) } \\
& \text { Suit une glose détaillée car, précise l'auteur, } \\
& \text { les choses dessusdites, selon la verité, ne sont pas sans mistere, ainz sont de grant } \\
& \text { significacion, ja soit que ce samble fictions estre et fable sanz raison pour la plus } \\
& \text { grant partie (ibid.) }
\end{aligned}
$$

Reprenant une pratique habituelle de l'allégorèse, Evrart découpe alors en unités discrètes le texte à gloser et impose à chacune des parties dégagées un ou plusieurs sens, le commentaire étant introduit par des formules du type «nous pouvons aussi dire que ", " on pourrait aussi dire que, tout aussi que..." ", etc. Sont ainsi tour à tour «exposées» la ou les significations de la fontaine, de l'eau, du pin, de la pierre de marbre, des deux cristaux. Pour l'histoire de Narcisse et d'Echo, la technique utilisée, un peu différente, est également usuelle. «L'exposition» empile en effet plusieurs strates de significations possibles qui font successivement de Narcisse un jeune homme indifférent à l'amour qu'on lui porte (sens hérité), la victime des refus d'une «jouvencele» dont il tombe amoureux (et qui venge ainsi Echo), un homme trop sensible à la beauté de la femme aimée, la femme étant «come le ymage et similitude de l'homme » (p. 592), ou encore celui qui, peut-on dire, " aime son umbre et son ymage», parce qu'il aime celle « ou il ne peut trouver amour correspondant ne riens de ce que on desire en amours» (p. 592). Et, conclut (provisoirement) l'auteur, « ainsy peut-il estre assez ymaginable que Narcisus moru » (p.593), ajoutant encore que si la «fable faint » que Narcisse fut "transmués en fleur», cela signifie qu'il fut semblable à une fleur «dont la beauté est tost expiree et faillie » (la glose est déjà dans l'Ovide moralisé), et que «son ame fu en enfer translatee, c'est a dire en la terre, selon l'opinion d'aucuns des anciens qui disoient que les ames des mors y descendoient toutes» (p. 593), glose savante inconnue, elle, de l'Ovide moralisé.

Il faudrait analyser dans le détail le texte très touffu et le commentaire soigneusement médité d'Evrart de Conty. Mais on voit bien comment le projet d'ensemble de cette glose trop éclatée est de rationaliser autant que possible et non de "christianiser» le 
mythe, et moins encore de le moraliser au sens banal du terme. Il s'agit bien, pour Evrart, ce membre éminent de la Faculté de médecine de Paris ${ }^{25}$ en qui l'on verra volontiers le prédécesseur du Stendhal de De l'amour, de réorienter l'histoire de Narcisse vers le problème central du désir charnel en substituant à l'ombre vainement désirée 'l'image'd'une jeune fille, d'une belle dame sans merci que n'a pu séduire Narcisse et qui a causé sa mort. Narcisse devient ainsi le double masculin d'Echo, de la «jone pucelle qui ama Narcisus pour sa beauté, si forseneement que elle en moru finablement de deul, pour ce que cely n'avoit de s'amour cure» (p. 591) Mais l'auteur n'en reste pas là. Signalant lui-même qu'il va encore développer la glose, il prend prétexte de l'échec identique d'Echo et de Narcisse pour engager une très longue réflexion sur l'amour, sa nature «naturelle », les conditions de sa naissance (la vue, bien entendu) et s'interroger enfin sur ce mystère : comment peut-on analyser le sort de Narcisse (et d'Echo), comment expliquer la non réciprocité du désir? La partie se joue, pour résumer un très long débat, sur la notion clé de similitude, loi de la nature selon laquelle chaque élément de la création recherche la compagnie de son semblable et qui sous-tend par exemple les liens sociaux ou l'amour paternel. Quant aux similitudes elles-mêmes, elles seront d'autant plus fortes qu'elles seront plus «natureles, sy come estre semblable en meurs et en complexion; et par especial, la complexion naturele vaint en cesti cas toutes les autres choses, car de la complexion mesmez se despendent les meurs et les inclinacions de l'ame selon les philosophes. Et pour ce dit Aristote, sy come il a esté dit devant pluseurs foiz, que 'l'ame ensieut le corps tant que elle peut', c'est a dire que la disposicion de l'ame quant a ses natureles inclinacions, ensieut la disposicion du corps et la complexion ».(pp.598-599). Mais il en va de la chaîne d'or qui lie ou non les êtres comme de la plus ou moins grande concordance des sons :

aussi que on voit es sons qui sont consonans l'un a l'autre, sy come on pourroit dire que a. b. c. seroient troiz sons dont .b. et .a. ensamble comparé feroient la consonancie qui est en musique appelle dyapente, c'est a dire une quinte, et .c. et .a. en feroient une autre appelle dyapason, c'est a dire une double;.b. donc se acorderoit a .a. et .a. aussi se acorderoit a .b. , maiz .a. et .c. se acorderoient encore mielx ensamble, pour ce que la double proporcion fait la meilleur consonancie et la plus doulce qui puisse estre et en laquelle les sons mielx se conforment come il a esté dit[...] Sanz faille, on pourroit dire que raison y fait bien aucune chose, qui doit regler aucunement l'amour en nostre espece humaine. Et pour ce y peut il bien estre que, avecques les natureles inclinacions dessusdites anclinans a amour pour les diverses consideracions des deux personnes, que il souffira en l'un ce qu'il verra en l'autre; et par ainsy, il s'y arrestera et ly donrra s'amour entierement. Mais ce que ly autres verra en ly ne ly souffira pareillement espoir, ainz ly souffira mielx par avanture ce qu'il verra en une autre, sy s'y arrestera pour la plus grant plaisance.......] Ce n'est mie merveilles donc se Narcisus ne voult amer Echo, maiz il ama son umbre, come la fable faint (pp. 601-2).

La démonstration qui met en tension, à partir du cas Narcisse et de l'image reflétée par la fontaine, la nécessaire similitude ou concordance de deux êtres comme fondement naturel à l'amour et la cristallisation toujours imprévisible du désir charnel, est sans doute très laborieuse. Du moins montre-t-elle comment, avant de connaître une durable éclipse, le mythe de Narcisse a pu échapper à une moralisation banalisée pour formuler cette inquiétante énigme: l'impossibilité de fonder en raison l'attirance sexuelle, cette pulsion naturelle qu'Evrart désigne du mot clé de «plaisance ». amoureuse de Guillaume de Machaut ${ }^{26}$, composée peu auparavant, Narcisse, toujours vif, 
n'est plus cependant qu'une splendide "ymage » sculptée par Pygmalion sur le grand pilier d'ivoire qui supporte une fontaine à laquelle se garderont bien de boire le sage poète et le chevalier amant?

\section{NOTES}

1.Voir Christine de Pizan, Epistre Othea, éd. critique par G. Parussa, Droz, 1999. Il s'agit de la séquence XVI.

2.Voir par exemple J. Frappier, «Variations sur le thème du miroir, de Bernard de Ventadour à Maurice Scève » dans Histoires, mythes et symboles, Genève, Droz, 1976, p. 149-167, M.-N. Toury, Mort et Fin'Amor dans la poésie d'oc et d'oil aux XII ${ }^{e}$ et XIII ${ }^{e}$ siècles, Paris, Champion, 2001, p. 283-295 et, plus généralement, L. Vinge, The Narcissus Theme in Western European Literature up to the early $19^{\text {th }}$ Century, Lund, 1967.

3.Sur le mythe de Narcisse et sur celui d'Echo, qui lui est lié, on consultera notamment les ouvrages de J. Fabre-Serris, Mythe et poésie dans les «Métamorphoses» d'Ovide. Fonctions et significations de la mythologie dans la Rome augustéenne, Klincksieck, 1995 ; V. GélyGhedira, La nostalgie du moi. Echo dans la littérature européenne, PUF, 2000 ; J. Kristeva, Histoires d'amour, Denoël, «L'infini », 1983 ; P. Maréchaux, Énigmes romaines, Gallimard, «Le Promeneur », 2000 ; Narcisses, sous la direction de J. B. Pontalis, Gallimard, 1976, «Folio essais », 2000.

4.J. Frappier, art. cit., p. 154.

5.Selon l'expression de J.-P. Néraudau dans sa préface aux Métamorphoses, Gallimard, «Folio classique », 1992, p. 9. Toutes les citations d'Ovide renvoient à cette traduction. 6.Les citations renvoient à notre édition bilingue, Pyrame et Thisbé, Narcisse, Philomena, Gallimard, «Folio classique », 2000.

7.Ce sera là l'apport des contes tirés du folklore celtique et l'on sait les liens qu'on peut tisser entre Narcisse et le Guigemar des Lais de Marie de France, par exemple.

8.Benoît de Sainte-Maure, Le Roman de Troie, éd. bilingue (extraits) par E. Baumgartner et F. Vielliard, Paris, Le livre de poche, Lettres gothiques, 1998. Benoît unit ici des passages du récit ovidien sur Narcisse (la chasse, la soif) à une description de la «fontaine » tirée de l'Héroïde XVI, dans laquelle Pâris, rappelons-le, relate à Hélène la scène du jugement.

9.Paul Valéry, Fragments du Narcisse. 10.Voir Cligès, éd. bilingue par Ch. Méla et O. Collet, Le Livre de Poche, Lettres gothiques, 1994, vv. 2720-2725.

11.Les citations renvoient à l'éd. F. Lecoy, Guillaume de Lorris et Jean de Meun, Le Roman de la Rose, Champion, CFMA, 3 vol. 1965-1970.

12.Voir éd. cit. vv. 1521 et ss.

13.Même si Pygmalion se déclare moins fou que Narcisse, puisque lui au moins peut embrasser l'objet de son désir (éd. cit. vv. 20848- 20858).

14.Les citations renvoient à l'éd. C. de Boer, Amsterdam, Müller, 5 vol., 1915-1936. La fable de Narcisse et sa moralisation se lisent aux vv. 1301- 1964 du Livre III. Sur la 
réception d'Ovide dans l'Ovide moralisé, voir R. Blumenfeld-Kosisnki, Reading Myth. Classical Mythology and Its Interpretations in Medieval French Literature, Stanford University Press, 1997, Chap. 3. Sur l'Ovide moralisé lui-même, on se reportera à l'ouvrage pionnier de P. Demats, Fabula, Droz, 1973 et plus récemment à M.-R. Jung, « Aspects de l'Ovide moralisé » dans Ovidius redivivus, éds. M. Picone et B. Zimmermann, Stuttgart, 1994, p. 149-172 et du même auteur, "Ovide, texte, translateur et gloses dans les manuscrits de l'Ovide Moralisé ", The Medieval Opus: Imitation, Rewriting and transmission in the French Tradition, éd. D. Kelly, Amsterdam/Atlanta, Rodopi, 1996, p. 75-98. Voir aussi J.-Y. Tilliette, «L'écriture et sa métaphore. Remarques sur l'Ovide moralisé », dans Mélanges M.-R. Jung, éd. L. Rossi, Edizioni dell'Orso, 2 vol., 1996, p. 543-558.

15.A titre d'exemple les vv. 1569-1570 c'est li miroërs perilleus/ ou Narcisus li orgueilleus... deviennent pour les besoins de la glose : C'est li mireoirs perillous/ ou se mirent li orgueillous (L. III, vv. 1925-1926 de l'éd. De Boer).

16.Je ne sais si l'auteur a inventé ou, ce qui est plus probable, récupéré à une glose antérieure la mention de la ville de 'Narci'. Faut-il mettre ce nom en relation avec la ville de Narycie, ville du Bruttium (de la Calabre) citée au L. XV, v. 705 des Métamorphoses dans l'épisode consacré à Esculape?

17.Plusieurs des contributions ici réunies concernent d'ailleurs la réception de l'Ovide moralisé aux XIV et $\mathrm{XV}^{\mathrm{e}}$ siècles. Sur l'usage à partir du XIV siècle des «fictions " ovidiennes, voir la thèse de Didier Lechat, à paraître dans les Études christiniennes, éd. H. Champion.

18.Les citations renvoient, pour le texte en vers, à l'édition partielle de C. Kraft, Liebesgarten-Allegorie der Échecs amoureux, Francfort-sur-le-Main, 1977 et pour le texte en prose à l'éd. de F. Guichard-Tesson et B. Roy, Édition CERES, Montréal, 1993. Les deux textes ont fait l'objet d'une première étude pionnière par P.-Y. Badel dans Le Roman de la Rose au XIV siècle. Étude de la réception de l'œuvre, Genève, Droz, 1980. On se reportera pour la bibliographie récente à l'éd. F. Guichard-Tesson et B. Roy.

19.Ils restent en effet responsables de la mort de Narcisse, car ils sont «li mirëoirs merveilleus/ Ou Narchisus li orgueilleus/ Se mira tant, qu'il se trahy,/ Ainsy que vous avés oÿ ». (vv. 807-810)

20.Le héros aura en effet la possibilité d'engager une seconde partie (sa revanche ?) contre la belle jeune fille qui l'a une première fois "maté », mais la fin du texte manque. 21.Ouvr. cit., p. 281-282.

22.Voir Benoît de Sainte-Maure, Chronique des ducs de Normandie, éd. C. Fahlin, vv. 13348 et ss.

23.Même si se tisse un premier lien, comme on l'a vu plus haut, dans le Roman de Troie avec le récit du jugement de Pâris. Et il va de soi que le Roman de la Rose de Jean de Meun fait le même choix, mais en opposant plus précisément la statue de Pygmalion à «l'ymage » que vise crûment la flèche de Vénus.

24.Voir éd. cit., p. 418. La liste se poursuit avec dans l'ordre les exemples de Pasiphaé, Myrrha, Ménéphron, Phèdre, Térée, Scylla, Médée, Pyllis (abandonnée par Démophoon, exemple repris à Jean de Meun, v. 13181, et au delà à Héroïde II), Didon, Pyrame et Thisbé.

25.Voir les indications et la bibliographie données en pp. LIV-LV de l'éd. cit. 26.Guillaume de Machaut, La Fontaine amoureuse, éd. J. Cerquiglini-Toulet, Stock/Moyen Âge, 1993. Voir les vv. 1299-1312. 


\section{AUTEUR}

EMMANUĖLE BAUMGARTNER

Sorbonne nouvelle 